\title{
ADVANCES IN QUANTUM DOT INTERMEDIATE BAND SOLAR CELLS
}

\author{
E. Antolín ${ }^{1}$, A. Martî ${ }^{1}$, P. G. Linares ${ }^{1}$, I. Ramiro ${ }^{1}$, E. Hernández ${ }^{1}$, \\ C. D. Farmer ${ }^{2}$, C. R. Stanley ${ }^{2}$, and A. Luque ${ }^{1}$ \\ ${ }^{1}$ Instituto de Energía Solar, E.T.S.I. Telecomunicación, Universidad Politécnica de Madrid, 28040 Madrid, Spain \\ (E-mail: elisa@ies-def.upm.es) \\ ${ }^{2}$ Department of Electronics and Electrical Engineering, University of Glasgow, Glasgow G12 8QQ, U.K.
}

\begin{abstract}
Several groups have reported on intermediate band solar cells (IBSC) fabricated with InAs/GaAs quantum dots (QD) which exhibit quantum efficiencies (QE) for sub-bandgap photon energies. However, this QE is produced by the absorption of photons only through valence band (VB) to intermediate band (IB) transitions. The absorption of photons of that energy in IB to conduction band (CB) transitions is weak and is usually replaced by carrier escape. This mechanism is incompatible with the preservation of the output voltage, and therefore, it cannot lead to the high efficiencies predicted by the IBSC model. In this work, we discuss the contribution of thermal and tunneling mechanisms to IB-CB carrier escape in current QD-IBSCs. It is experimentally demonstrated that in QDIBSC prototypes where tunnel escape has been eliminated, the sub-bandgap $\mathrm{QE}$ is suppressed at sufficiently low temperatures, and when this occurs, the only limit for the open-circuit voltage $\left(V_{O C}\right)$ is the fundamental semiconductor bandgap, as stated by the IBSC theoretical model.
\end{abstract}

\section{INTRODUCTION}

The IBSC concept has been described extensively in previous works [1, 2]. Its ideal operation is illustrated by the band diagram in Figure 1. The existence of an isolated IB within the fundamental bandgap $\left(E_{\mathrm{G}}\right)$ of the IB material enables the absorption of low energy photons through the transitions labeled (1) and (2) (across the sub-bandgaps denoted $E_{\mathrm{H}}$ and $E_{\mathrm{L}}$, respectively). As the absorption of high energy photons is still possible through transition (3), the cell is capable of producing extra photocurrent with respect to a conventional cell with gap $E_{\mathrm{G}}$. Very high photovoltaic conversion efficiencies can be achieved because this enhanced photocurrent can be extracted without significant degradation of the output voltage. The voltage at the contacts is only limited by $E_{\mathrm{G}}$ - as it would be in the case a conventional cell with that gap - and not by the lower absorption thresholds $E_{\mathrm{H}}$ and $E_{\mathrm{L}}$. In the implementation with InAs/GaAs QDs the IB arises from the confined electron levels in the QDs [3]. A simplified band diagram for this case, under similar conditions to that Figure 1, can be seen in Figure 5 (b).

The first fabricated QD-IBSC prototypes were reported in 2004 [4]. Since then, several research groups have shown important advances in the implementation of QD-IBSCs based on the InAs/GaAs QD material system. In particular, it had been realized that the accumulation of strain in the QD stack can lead to the formation of dislocations, which degrades the photocurrent collection [5]. The application of strain compensating growth techniques - either through the insertion of $P$ [6] or N [7] in the GaAs barrier material of the QD stack - has enabled the production of QDIBSCs that exhibit significantly more photocurrent than $\mathrm{GaAs}$ reference cells of similar characteristics. Following an alternative growth method, an efficiency of $18 \%$ has been demonstrated [8] (here the In content in a (In, Ga)As/GaAs QD matrix is varied as the number of layers is increased).

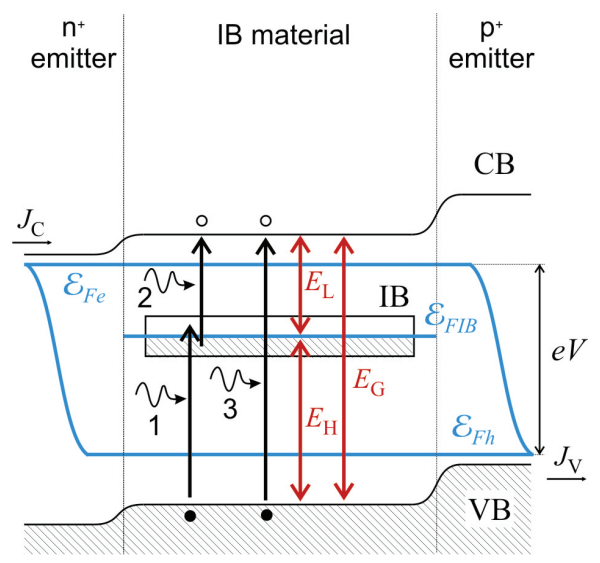

Figure 1: Band diagram of an IBSC under illumination and positive bias. $E_{\mathrm{G}}$ is the fundamental bandgap, $E_{\mathrm{L}}$ and $E_{\mathrm{H}}$ the sub-bandgaps; $\varepsilon_{\mathrm{FIB}}, \varepsilon_{\mathrm{Fe}}$ and $\varepsilon_{\mathrm{Fh}}$ are the quasi-Fermi levels associated with the IB, CB and VB, respectively. In the ideal case, the external voltage, $V$, equals the $\left(\varepsilon_{\mathrm{Fe}}-\varepsilon_{\mathrm{Fh}}\right)$ split (divided by the electron charge, $e$ ) and, thus, it s only limited by $E_{\mathrm{G}}$.

However, the eventual achievement of really high efficiencies in future QD-IBSC prototypes depends on the preservation of the voltage. This characteristic makes the QD-IBSC proposal fundamentally different from other solar cells in which quantum structures are implemented with the aim of tuning the absorption threshold, such as quantum well solar cells (QWSC) [9]. For the moment, all InAs/GaAs QD-IBSCs reported exhibit some reduction of the $V_{O C}$ with respect to $\mathrm{GaAs}$ reference cells at room temperature. Recently, very small differences in $V_{O C}$ have been demonstrated (39 meV in [10]). But, as it will be discussed later on, this does not constitute a conclusive 
proof of the principle of voltage preservation. In this work we demonstrate voltage preservation on QD-IBSCs by fundamental experiments at low temperatures. This principle is intrinsically related to the mechanism of subbandgap photocurrent production, and therefore, our analysis shall start from the interpretation of $Q E$ experiments.

\section{INTERPRETATION OF PREVIOUS RESULTS: RELATION BETWEEN QUANTUM EFFICIENCY AND VOLTAGE PRESERVATION}

Figure 2 shows the measured $Q E$ of a standard InAs/GaAs QD-IBSC (sample S1). It contains 10 layers of InAs QDs separated by $10 \mathrm{~nm}$ thick, Si $\delta$-doped GaAs spacers. The $\delta$-doping with donors aims to half-fill the IB to promote absorption in the IB $\rightarrow \mathrm{CB}$ transition [11]. As all the samples presented here, it has been grown by solid source molecular beam epitaxy (MBE), the QDs being formed under the Stranski-Krastanov growth mode [12]. Fabrication details of similar samples can be found in our previous works [4]. The peaks observed in the subbandgap QE of the QD-IBSC indicate the energy of interband transitions between confined states, as illustrated on the simplified band diagram on the right [13]. The peak labeled (a) can be related to transitions between confined states of the wetting layer (WL), (d) arises from transitions between the ground-states of the dots and further peaks (b, c) from transitions between excited states. The energy of peak (d) is identified with $E_{\mathrm{H}}$ in the QD-IBSC description (the presence of excited sates is not favourable for the IBSC concept but their effect can be borne in some cases [14]). Taking into account the reduction of the effective $E_{G}$ due to the presence of the WL and the VB offset (discussed elsewhere [15]), the sub-bandgaps of this QDIBSC are $E_{\mathrm{H}}=1.053 \mathrm{eV}$ and $E_{\mathrm{L}} \sim 0.2 \mathrm{eV}$. This QE is qualitatively representative of most QD-IBSCs reported to date, although the $E_{H}$ value is higher in many cases $[6,10$, $16,17]$.

There are important differences between the absorption properties of QD inter-band transitions (VB-IB transitions in the QD-IBSC context) and intra-band transitions (IB-CB transitions). In fact, in the QE experiment only the first ones take place. The experimental results in the next section will demonstrate this statement; here we review its theoretical justification. It has been shown that only the intra-band transitions between contiguous confined states (small difference in quantum number) have reasonably high oscillator strengths [18]. Due to strain-induced effects, practical InAs/GaAs QDs show high aspect ratio (elongated base, reduced height) which results in the appearance of many electron levels for low values of $E_{\mathrm{L}}$. Those levels are closely spaced in energies (for example, the difference between peaks (c) and (d) in Figure 2 is 85 meV for $E_{\mathrm{L}} \sim 0.2 \mathrm{eV}$ ). Therefore, the absorption in IB-CB transitions becomes only important for photon energies in that range, much lower than the range utilized in the $Q E$ experiment. Since it is not plausible that the absorption in
IB-CB transitions of photons of more than $1.0 \mathrm{eV}$ contributes to the measured $\mathrm{QE}$, the extraction of subbandgap photocurrent has to be attributed to the optical transition of carriers from the VB to IB, and subsequent non-optical carrier escape from the IB to the $C B$ (see Figure 2, right) .

It has to be noted that in the IBSC model, voltage preservation is possible because when an electron-hole pair is produced by the absorption of two sub-bandgap photons, the free energy of both photons is utilized to produce electric work. It can be demonstrated on the base of thermodynamic arguments [19] that the efficiency limit in the case where only one sub-bandgap optical transition is operative (and the other supplanted, for example, by thermal escape) cannot surpass the limit of single gap cells because the voltage is not preserved. Therefore, in an ideal QD-IBSC with the sub-bandgap absorption properties of the $\ln A s / G a A s$ QD material system the measured sub-bandgap QE should be zero. A result as the one shown in Figure 2 indicates strong IB-CB carrier escape, which hampers the quasi-Fermi level split between the $\mathrm{CB}$ and IB, and thus, makes strict voltage preservation impossible.



Figure 2: Left: external QE of QD-IBSC $S 1$ in the subbandgap range (solid line) compared with a GaAs reference cell (dashed line). Right: simplified band diagram of an InAs/GaAs QD indicating the possible transitions originating the peaks observed in the $Q E$.

The feasibility of producing two-photon photocurrent has been demonstrated in InAs/GaAs QD-IBSCs using low energy photons to excite the IB-CB transition [20]. However, the effect is weak and could only be observed at low temperatures, when the IB-CB thermal escape is reduced [21]. It can be concluded from this analysis that, to pursue voltage preservation in the QD-IBSC, the optical absorption in the IB-CB transition has to be promoted, while all other mechanisms for IB-CB carrier escape have to be reduced. It has been proposed that the improvement of the QD aspect ratio, which should lead to a lower 
number of electron confined states separated by higher energy splits, can serve to both purposes [18, 22].

\section{REDUCING IB-CB CARRIER ESCAPE}

Two QD-IBSC samples (S2 and S3) have been fabricated with improved characteristics with respect to sample S1: - Sample S2 contains 11 layers of InAs QDs, which have been capped with InAIGaAs strain relief layers (SRL) with the aim of improving the aspect ratio of the dots [22]. The QD layers are separated by $13 \mathrm{~nm}$ thick GaAs spacers, and field damping layers (FDL) have been inserted between the QD stack and the emitters, which were not present in sample S1. The FDLs prevent the tunneling of carriers from the $I B$ to the $C B$ at the junction with the emitter [23].

- Sample S3 contains 30 layers on InAs QDs, capped by similar InAIGaAs SRLs and separated by $84 \mathrm{~nm}$ thick GaAs spacers.

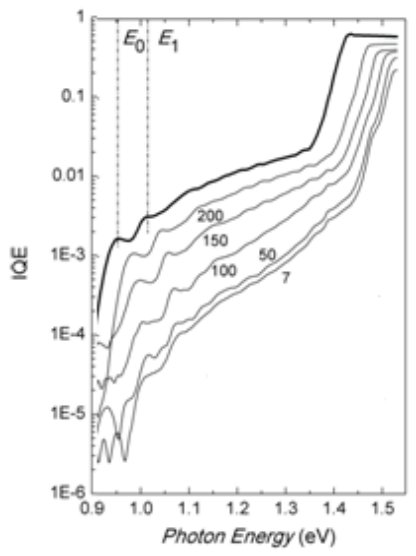

(a) Sample S2

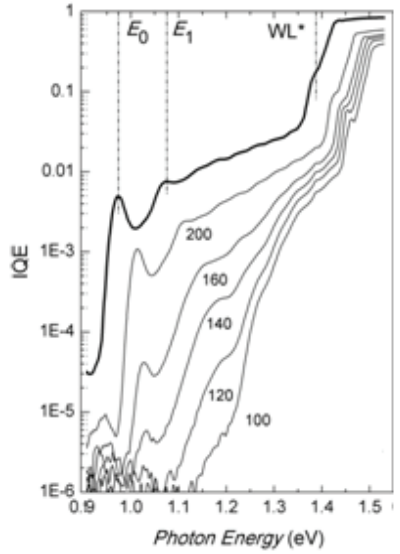

(b) Sample S3

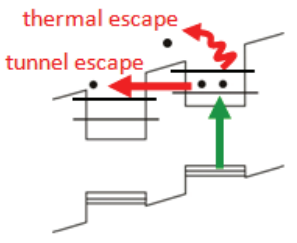

Figure 3: Internal QE of samples S2 (a) and S3 (b). The thick lines in each plot represent the measurements taken at room temperature and the other lines correspond each to the temperature indicated by its label (in K). The graphs on the right show the possible IB-CB escape mechanisms for each sample, as discussed in the text.
Figure 3 shows the internal quantum efficiency (IQE) measured at different temperatures on samples S2 (a) and S3 (b). They exhibit different behaviors at low temperature. The sub-bandgap QE in sample $\mathrm{S} 2$ is reduced as $T$ is decreased, but it is not suppressed, not even for values as low as $7 \mathrm{~K}$. A similar behavior at low temperatures has been found for other InAs/GaAs QDIBSC prototypes [24]. Contrarily, in sample S3 the subbandgap $Q E$ vanishes for temperatures below $120 \mathrm{~K}$. The diagrams on the right illustrate plausible IB-CB carrier escape mechanisms that explain those results. In the case of S2 some not thermally activated (i.e. tunneling) escape must be present. Since this sample contains FDLs, the tunneling of IB carriers to the $\mathrm{CB}$ at the junction with the emitter is ruled out. Therefore, tunneling processes have to take place within the QD stack, for example, resonant tunneling from the ground-state of one dot through the GaAs spacer to excited states of the next dot, and from there to the barrier. The results for sample S3 show that the presence of thick GaAs spacers eliminates this possibility and the IB-CB carrier escape is here of purely thermal nature. Two conclusions can be drawn from the IQE of sample S3: (a) the sub-bandgap QE does not result from the absorption of two photons, as anticipated in the previous section; (b) it is possible to achieve a zero subbandgap QE, as expected from a proper IBSC behavior, if tunneling escape is eliminated. The temperature at which QE suppression takes place depends on the activation energy of thermal escape. In the case of sample S3 the activation energy is $224 \mathrm{meV}$. For conventional, GaAs capped, InAs dots, we have measured an activation energy of $115 \mathrm{meV}$ and the sub-bandgap QE is only suppressed at temperatures well below $100 \mathrm{~K}$. The effects of the InAIGaAs SRL which are responsible for the reduction in the strength of thermal escape in sample S3 are discussed in [22]

\section{DEMONSTRATION OF THE VOLTAGE PRESERVATION PRINCIPLE IN THE QD-IBSC}

It is apparently straightforward to test voltage preservation by comparing the $V_{\mathrm{OC}}$ of the fabricated QD-IBSCs with that of a GaAs reference cell. However, one might find, at least, three difficulties in the interpretation of results. In the first place, if the technology of QD growth is not mature, the $V_{O C}$ at room temperature can be degraded by parasitic problems (such as non radiative recombination due to defects), leading to incorrect conclusions about the feasibility of the QD-IBSC concept. Secondly, the principle of voltage preservation does not imply, even in the ideal case (radiative limit), that the $V_{O C}$ of the QD-IBSC equals that of the reference cell. According to detailed balance calculations, the difference in $V_{O C}$ between the IBSC and a single gap cell of same gap $E_{\mathrm{G}}$ is negligible for optimum values of $E_{\mathrm{G}}, E_{\mathrm{H}}$ and $E_{\mathrm{L}}$ under maximum concentration [13]. But the situation can be different for other cases. For instance, for the gaps of the InAs/GaAs QD system, which are far from optimal, it has been calculated that in the radiative limit the $V_{O C}$ at one sun is significantly lower than 
that of a GaAs cell [23] (the difference is reduced as to produce an efficiency exceeding that of the GaAs cell only for concentrations of 1000 suns or greater).

The third problem is that the $V_{O C}$ of the fabricated reference cell is not necessarily the highest achievable value. For example, a recent paper [10] reports $V_{O C}$ values as high as $0.873 \mathrm{~V}$ for InAs/GaAs QD-IBSCs, to be compared with $0.912 \mathrm{~V}$ for a GaAs reference. In fact, this difference is very small, although not as much if the $V_{O C}$ is compared with the most efficient GaAs cells under one sun illumination (presently over $1 \mathrm{~V}$ [25]). As the authors claim, this result is positive in the context of solar cells under the single gap cell efficiency limit, such as QWSCs, but it does not give further insight into the potential of QDs to implement an IBSC. It has to be taken into account that the sub-bandgap absorption threshold $\left(E_{\mathrm{H}}\right)$ of those QDIBSCs is $\sim 1.2 \mathrm{eV}$. Therefore, it cannot be discriminated whether the $V_{O C}$ of the QD-IBSC is indeed limited by $E_{G}$ or by $E_{\mathrm{H}}$. If the later is true, any attempt to reduce $E_{\mathrm{H}}$ in order to enhance the sub-bandgap photocurrent production would lower the Voc.

A conclusive way of determining the limit for the $V_{O C}$ of the QD-IBSC is to make the $V_{\mathrm{OC}}$ approach that limit by lowering the operation temperature. Figure 4 shows the dependence on $T$ of the $V_{O C}$ measured from (a) a GaAs reference cell, (b) QD-IBSC S1 and (c) QD-IBSC S3. A HeNe laser $(\lambda=632.5 \mathrm{~nm})$ was used to illuminate the samples. The Voc's are compared at each temperature with the GaAs gap (black line) and the value of $E_{\mathrm{H}}$ (red line), extracted from QE measurements such as those in Figure 3.

For the GaAs reference cell, the $V_{O C}$ approaches $E_{G}$ (when expressed in $\mathrm{eV}$ ) at very low temperatures, as expected from the classical theory of single gap solar cells [26] (the power density in the measurement shown is $\sim 0.1$ suns). Contrarily, in the case of sample $\mathrm{S} 1$ the $V_{\mathrm{OC}}$ cannot surpass $1.2 \mathrm{~V}$. We have included in this case the measurement under different illumination densities to corroborate that the result is not a consequence of insufficient illumination. $\mathrm{HI}$ corresponds to $\sim 10$ suns illumination, $\mathrm{Ml}$ to $\sim 1$ sun and $\mathrm{LI}$ to $\sim 0.1$ suns. It can be seen that $1.2 \mathrm{~V}$ is the real limit because all curves saturate at almost the same voltage. The sub-bandgap QE of this sample is not suppressed at low temperatures (not shown). Following the argumentations given in the previous section, we conclude that this sample does not fulfill voltage preservation because of the existence of tunneling mechanisms that connect the IB and the $C B$, preventing the corresponding quasi-Fermi level split [see Figure 5 (a)]. Since this sample does not include FDLs, tunneling is possible not only within the QD stack, as discussed for sample S2, but also at the junction with the emitter. Interestingly, the limit found for the $V_{O C}$ does not coincide with $E_{\mathrm{H}}$ (the energy of the ground-state transition peak in the $Q E$, red line) but with the energy of the firstexcited-state transition peak (blue line). This may indicate that at low temperatures the ground-state is actually disconnected from the $\mathrm{CB}$ and tunneling takes place only from QD excited states.

(a)

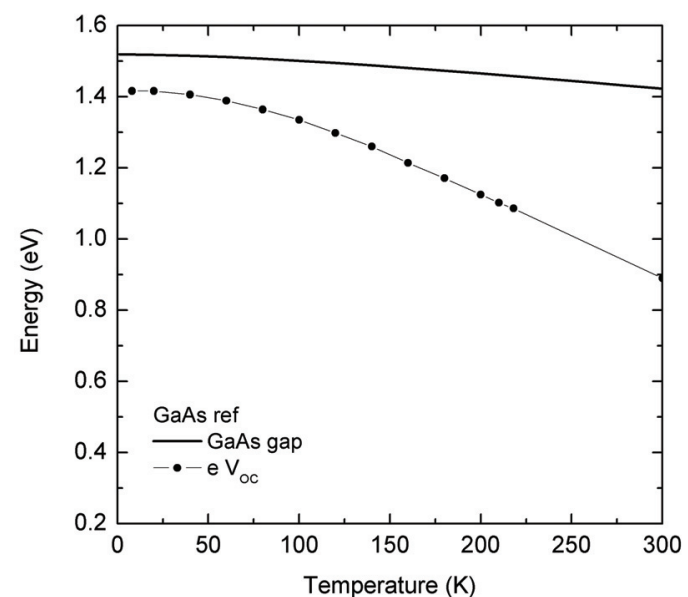

(b)

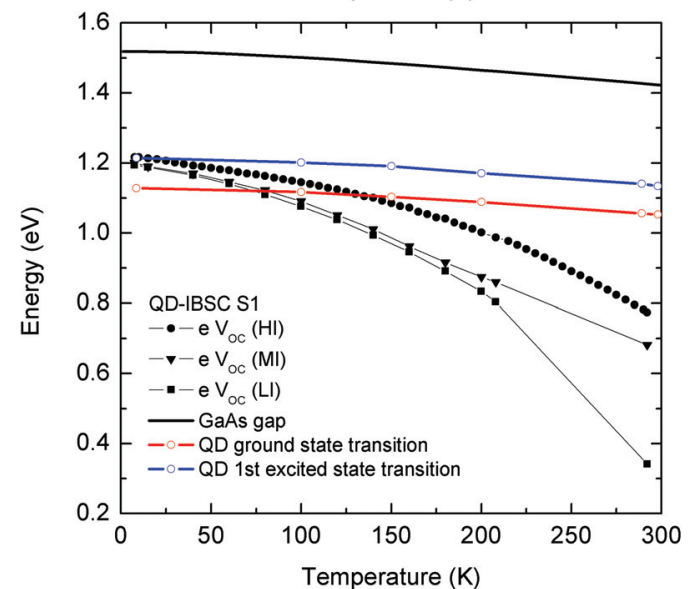

(c)

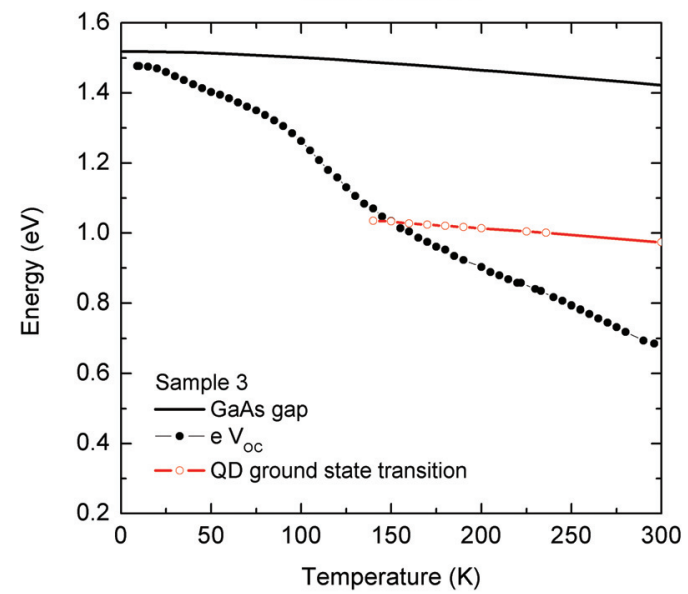

Figure 4: Comparison between $V_{O C}$ and absorption threshold at different temperatures for: (a) a GaAs reference cell, (b) QD-IBSC S1, and (c) QD-IBSCs S3 (symbols are measured data and lines are a guide to the eye). 
The measurement on sample S3 [Figure 4(c)] shows that the $V_{O C}$ increases over $E_{H}$ and is only limited by $E_{\mathrm{G}}$. This is, to our knowledge, the first empirical demonstration of the IBSC principle of voltage preservation on a QD-IBSC prototype. This result is in agreement with the analysis of the $\mathrm{QE}$, in which it was concluded that the thick GaAs spacers implemented in sample S3 prevent IB-CB tunnel escape [Figure 5 (b)]. The $E_{\mathrm{H}}$ values (red line) could only be determined for $T>120 \mathrm{~K}$ because at lower temperatures the sub-bandgap $\mathrm{QE}$ is suppressed. $V_{\mathrm{OC}}=1$ $\mathrm{V}$ is reached at $160 \mathrm{~K}$ (the power density in this measurement is $\sim 1$ sun). In fact, the highest $V_{\text {oc }}$ value $(1.477 \mathrm{~V})$ achieved at the lowest measured temperature (9K) exceeds the highest value measured for the reference cell $(1.416 \mathrm{~V})$. The same has been observed using other QD-IBSC and reference samples under different power densities and the reason is still unknown.

(a)

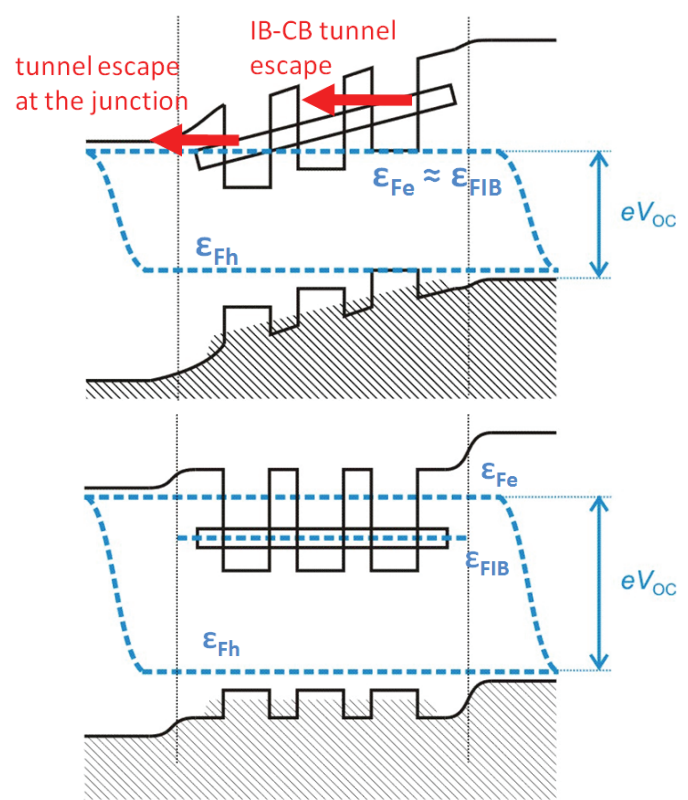

Figure 5: Simplified band diagrams (assuming flat quasi-Fermi levels) for QD-IBSC prototypes under open-circuit conditions: (a) does not preserve the $V_{\text {OC }}$ due to IB-CB tunnel escape; (b) behaves as an IBSC, preserving the $V_{O C}$.

In addition to providing a proof of the concept, these experiments can be used to illustrate future paths of improvement for practical QD-IBSCs. The strategy applied here, increasing the spacer thickness, has proven to eliminate IB-CB tunneling. But it is also possible that similar results can be obtained with QD-IBSCs with thin spacers and adequate design (FDLs, QDs with well separated excited states). At this respect, it has to be remembered that the electric field on the dots in a $Q E$ experiment (short-circuit) is higher than under operation conditions. On the other hand, other strategies besides the use of InAIGaAs SRLs have to be investigated in order to further weaken thermal escape and shift the high $V_{O C}$ range to higher temperatures for the same or lower $E_{\mathrm{H}}$ values. Given the strong limitations found in the InAs/GaAs QD material system (see band diagram on Figure 2), these strategies should probably include the search for alternative QD material systems [27].

\section{CONCLUSIONS}

The principle of voltage preservation of the IBSC model has been demonstrated in low temperature experiments on samples fabricated with InAs/GaAs QDs. It has been shown that the $V_{O C}$ is only limited by the GaAs gap and not by the sub-bandgaps introduced by the IB. In particular, it could be demonstrated on samples with thick GaAs spacers between QD layers, because in them, IBCB tunneling carrier escape mechanisms have been eliminated. This is corroborated by the fact that the subbandgap quantum efficiency is suppressed at low temperatures. The relation between IB-CB carrier escape, sub-bandgap quantum efficiency and the attainment of voltage preservation in practical QD-IBSCs has been discussed.

\section{ACKNOWLEDGEMENTS}

This work has been supported by the IBPOWER project funded by the European Commission (Grant Agreement No. 211640), by the Regional Government of Madrid within the project NUMANCIA2 (S2009/ENE-1477), by the Spanish National Research Program within the project Consolider GENESIS-FV (CSD2006-0004) and by MICINN (project NANOGEFES ENE2009-14481-C02-02).

\section{REFERENCES}

[1] A. Luque and A. Martí, "Increasing the efficiency of ideal solar cells by photon induced transitions at intermediate levels," Phys. Rev. Lett. 78, pp. 5014-5017, 1997.

[2] A. Luque and A. Martí, "A metallic intermediate band high efficiency solar cell," Prog. Photovolt: Res. Appl. 9, pp. 73-86, 2001.

[3] A. Martí, L. Cuadra, and A. Luque, "Quantum dot intermediate band solar cell," in Conference Record of the 28th IEEE Photovoltaics Specialists Conference, New York, USA, 2000, pp. 940-943.

[4] A. Luque, A. Martí, C. Stanley, et al., "General equivalent circuit for intermediate band devices: potentials, currents and electroluminescence," J. Appl. Phys. 96, pp. 903-909, 2004.

[5] A. Martí, N. López, E. Antolín, et al., "Emitter degradation in quantum dot intermediate band solar cells," Appl. Phys. Lett. 90, p. 233510, 2007.

[6] S. M. Hubbard, C. G. Bailey, C. D. Cress, et al., "Short circuit current enhancement of GaAs solar cells using 
strain compensated InAs quantum dots " in Conference record of the 33rd IEEE Photovoltaic Specialists Conference San Diego, USA: IEEE, 2008.

[7] R. Oshima, A. Takata, and Y. Okada, "Straincompensated InAs/GaNAs quantum dots for use in highefficiency solar cells," Appl. Phys. Lett. 93, p. 083111, 2008.

[8] S. A. Blokhin, A. V. Sakharov, A. M. Nadtochy, et al., "AIGaAs/GaAs photovoltaic cells with InGaAs quantum dot arrays," Physics and Semiconductor Technique. 43, pp. 537-542, 2009

[9] K. W. J. Barnham, I. Ballard, J. P. Connolly, et al., "Quantum well solar cells," Physica E: Low-dimensional Systems and Nanostructures. 14, pp. 27-36, 2002.

[10] D. Guimard, R. Morihara, D. Bordel, et al., "Fabrication of $\operatorname{InAs} / \mathrm{GaAs}$ quantum ot solar cells with enhanced photocurrent and without degradation of open circuit voltage," Appl. Phys. Lett. 96, p. 203507, 2010.

[11] A. Martí, L. Cuadra, and A. Luque, "Partial filling of a quantum dot intermediate band for solar cells," IEEE Trans. Electron Dev. 48, pp. 2394-2399, 2001.

[12] M. Sugawara, Self-assembled InGaAs/GaAs quantum dots vol. 60: Semiconductors and Semimetals, Academic Press, 1999.

[13] E. Antolín, PhD. Thesis, Insituto de Energía Solar, UPM, Madrid, 2010.

[14] A. Luque, P. G. Linares, E. Antolín, et al., "Multiple levels in intermediate band solar cells," Applied Physics Letters. 96, p. 013501, 2010.

[15] A. Martí, L. Cuadra, and A. Luque, "Intermediate Band Solar Cells," in Next Generation Photovoltaics: High Efficiency through Full Spectrum Utilization, A. Martí and A. Luque, Eds. Bristol: Institute of Physics Publishing, 2003, pp. 140-162.

[16] V. Popescu, G. Bester, M. C. Hanna, et al., "Theoretical and experimental examination of the intermediate-band concept for strain-balanced (In, Ga)As/Ga(As,P) quantum dot solar cells," Phys. Rev. B. 78, p. 205321, 2008.

[17] R. B. Laghumavarapu, M. El-Emawy, N. Nuntawong, et al., "Improved device performance of InAs/GaAs quantum dot solar cells with strain compensation layers," Appl. Phys. Lett. 91, p. 243115, 2007.

[18] A. Luque, A. Martí, E. Antolín, and P. García-Linares, "Intraband Absorption for Normal Illumination in Quantum Dot Intermediate Band Solar Cells," to be published in Solar Energy Materials and Solar Cells, 2010.

[19] A. Luque, A. Martí, and L. Cuadra, "Thermodynamic consistency of sub-bandgap absorbing solar cell proposals," IEEE Trans. Electron Dev. 48, pp. 2118-2124, 2001.

[20] A. Martí, E. Antolín, C. R. Stanley, et al., "Production of Photocurrent due to Intermediate-to-Conduction-Band Transitions: A Demonstration of a Key Operating Principle of the Intermediate-Band Solar Cell," Phys. Rev. Lett. 97, p. 247701, 2006.

[21] E. Antolín, A. Martí, P. G. Linares, et al., "Demonstration and analysis of the photocurrent produced by absorption of two sub-bandgap photons in a quantum dot intermediate band solar cell," in Proc. of the 23rd
European Photovoltaic Solar Energy Conference Valencia, Spain: WIP - Renewable Energies, 2008, pp. 5-10.

[22] E. Antolín, A. Martí, C. D. Farmer, et al., "Reducing carrier escape in the InAs/GaAs quantum dot intermediate band solar cell," to be published in J. Appl. Phys., 2010.

[23] A. Martí, E. Antolín, E. Cánovas, et al., "Elements of the design and analysis of quantum-dot intermediate band solar cells," Thin Solid Films. 516, pp. 6716-6722, 2007.

[24] C. D. Cress, S. M. Hubbard, C. Bailey, et al., "Thermal Dependence of Quantum Dot Solar Cells," in Mater. Res. Soc. Symp. Proc., Materials Research Society. vol. 1031 2008, pp. 1031-H13-19.

[25] M. A. Green, K. Emery, Y. Hishikawa, and W. Warta, "Solar Cell Efficiency Tables (Version 33)," Prog. Photovolt: Res. Appl. 17, pp. 85-94, 2009.

[26] W. Shockley and H. J. Queisser, "Detailed Balance Limit of Efficiency of p-n Junction Solar Cells," J. Appl. Phys. 32, pp. 510-519, 1961.

[27] A. Luque and A. Marti, "The intermediate band solar cell: progress towards the realization of an attractive concept," Advanced Materials. 22, pp. 160-174, 2009. 